\title{
Non-Markovian continuous-time quantum walks on lattices with dynamical noise
}

\author{
Claudia Benedetti, ${ }^{1, *}$ Fabrizio Buscemi ${ }^{2, \dagger}$ Paolo Bordone, $, 2,3, \ddagger$ and Matteo G. A. Paris ${ }^{1,4,5, \S}$ \\ ${ }^{1}$ Quantum Technology Lab, Dipartimento di Fisica, Università degli Studi di Milano, I-20133 Milano, Italy \\ ${ }^{2}$ Dipartimento di Scienze Fisiche, Informatiche e Matematiche, Università di Modena e Reggio Emilia, via Campi 213/A, \\ Modena I-41125, Italy \\ ${ }^{3}$ Centro S3, CNR-Istituto Nanoscienze, Via Campi 213/A, 41125 Modena, Italy \\ ${ }^{4}$ Istituto Nazionale di Fisica Nucleare, Sezione di Milano, I-20133, Milano, Italy \\ ${ }^{5}$ Consorzio Nazionale per la Fisica della Materia, Unità Milano Statale, I-20133 Milano, Italy
}

(Received 2 November 2015; published 12 April 2016)

\begin{abstract}
We address the dynamics of continuous-time quantum walks on one-dimensional disordered lattices inducing dynamical noise in the system. Noise is described as time-dependent fluctuations of the tunneling amplitudes between adjacent sites, and attention is focused on non-Gaussian telegraph noise, going beyond the usual assumption of fast Gaussian noise. We observe the emergence of two different dynamical behaviors for the walker, corresponding to two opposite noise regimes: slow noise (i.e., strong coupling with the environment) confines the walker into few lattice nodes, while fast noise (weak coupling) induces a transition between quantum and classical diffusion over the lattice. A phase transition between the two dynamical regimes may be observed by tuning the ratio between the autocorrelation time of the noise and the coupling between the walker and the external environment generating the noise. We also address the non-Markovianity of the quantum map by assessing its memory effects, as well as evaluating the information backflow to the system. Our results suggest that the non-Markovian character of the evolution is linked to the dynamical behavior in the slow noise regime, and that fast noise induces a Markovian dynamics for the walker.
\end{abstract}

DOI: 10.1103/PhysRevA.93.042313

Quantum walks (QW) are the quantum analog of the classical random walks [1,2] and describe the propagation of a quantum particle over an $n$-dimensional graph. Because of their quantum nature, which allows for quantum superposition of states and interference, QWs show a very different behavior compared to their classical counterparts. These features allow one to exploit QWs for tasks that cannot be achieved with the limited resources of classical random walks. Much interest has arisen around QWs especially because of their central role in nondeterministic algorithms [3], universal quantum computation [4], transport through a graph [5-7], and in modeling processes in biological systems [8-10]. The generalization of random walks to the quantum realm leads to two classes of QWs: discrete-time quantum walks (DTQWs), where the Hilbert space of the particle position is joined with the Hilbert space of a quantum coin [11], and continuous-time quantum walks (CTQWs), which operate only in the position space [12]. Both have been proved very efficient to speed up quantum algorithms compared to their classical counterparts, and experimental implementation schemes have been proposed in a variety of systems, both for DTQWs [13-16] and CTQWs [17,18]. The dynamics of a particle in discrete- and continuous-time QW shows similar features and a formal connection between the two classes of quantum walks has been proved in some regimes $[19,20]$.

In this paper, we study continuous-time quantum walks on a one-dimensional graph, such as a line or a circle, i.e., quantum version of continuous-time Markov chains [12,21,22].

\footnotetext{
*claudia.benedetti@unimi.it

†fabrizio.buscemi@unimore.it

${ }^{\ddagger}$ paolo.bordone@unimore.it

\$matteo.paris@fisica.unimi.it
}

CTQWs on the line are defined on a set of orthonormal position states $\{|j\rangle\}_{k=1}^{N}$, where $|j\rangle$ represent a localized state of the walker in the $j$ th node of a one-dimensional lattice and $N$ is the total number of graph sites. Due to the laws of quantum mechanics, the quantum walker may simultaneously occupy all the lattice nodes, with interference effects that allow the particle to propagate faster than in the classical version. In this paper, we will focus on CTQW where only nearest-neighbor transitions are allowed, i.e., the particle can jump (tunnel) only to the nearest sites. In this scenario, the particle Hamiltonian is the discrete Laplacian operator, i.e., the Hamiltonian describing the free evolution of a particle in a periodic potential:

$$
H_{0}|j\rangle=2|j\rangle-|j+1\rangle-|j-1\rangle \text {. }
$$

The eigenvectors $\left|\Psi_{\theta}\right\rangle$ and eigenvalues $E_{\theta}$ of the Hamiltonian (1) depend upon the choice of the boundary conditions. In the case of periodic boundary conditions, the solutions, found by adopting a Bloch function approach, take the expressions

$$
\begin{gathered}
\left|\Psi_{\theta_{n}}\right\rangle=\frac{1}{\sqrt{N}} \sum_{j=1}^{N} e^{-i \theta_{n} j}|j\rangle, \\
E_{\theta_{n}}=2-2 \cos \theta_{n},
\end{gathered}
$$

where $\theta_{n}=2 n \pi / N$ and $n \in[1, N]$. Notice, however, that our analysis is actually independent on the boundary conditions since we will confine ourselves to observe the dynamics of the walker before it reaches the borders of the graph.

Running the quantum walks for a time $t$ means applying the evolution operator $U(t)=e^{-i H t}$ to an initial state of the walker $\left|\psi_{0}\right\rangle$. Under the action of Hamiltonian (1), the quantum particle evolves with nonclassical propagation characteristics: for a localized initial state, the QW is distributed over the lattice 
nodes with a highly non-Gaussian probability distribution, showing two peaks that move away from the initial position as time increases. Moreover, the QWs spread more rapidly compared to classical random walks, with a variance $\sigma^{2}=$ $\left\langle x^{2}\right\rangle-\langle x\rangle^{2}$ proportional to $t^{2}$ (ballistic propagation) instead of the classical diffusive propagation $\sigma^{2} \propto t$.

The above modeling of CTQWs is an ideal description of the diffusion of a quantum particle over a perfect periodic potential, assuming that neither defects nor disorder in the lattice are present. However, in realistic physical implementations of QWs, noise is always present [23-27], due to fabrication imperfections or caused by the unavoidable interaction of the walker with the external environment, which induces decoherence. Decoherence, in turn, may either suppress the propagation of the walker wave function, leading to Anderson localization [28] which prevents the particle from spreading, or it may induce a transition from quantum to classical diffusion, thus changing the ballistic propagation of a quantum particle to a slower diffusive spreading and destroying the interference patterns that characterize QWs [29].

The effects of noise on discrete-time QWs received considerable attention [30-35], whereas an exhaustive analysis of the dynamics of noisy continuous-time QWs is still missing. As a matter of fact, the effects of static noise or of a single impurity in the lattice or of dynamical noise affecting the nodes energies have been analyzed by some authors [36-39]. However, full dynamical models of noise are needed in order to give a realistic description of quantum walks, suitable to describe the walker behavior in realistic conditions [40].

In this paper, we address the effects of noise induced by dynamical disorder on the behavior of a CTQW over a onedimensional discrete lattice. In order to describe dynamical disorder, we go beyond the Gaussian approximation and describe noise as non-Gaussian stochastic contribution to the tunneling amplitudes of the Hamiltonian. In fact, quantum features in the dynamics of a walker are mostly due to the presence of tunneling amplitudes in the interaction Hamiltonian (as opposite to tunneling probabilities of a classical walker) and thus adding noise to the off-diagonal elements of the Hamiltonian allows one to assess the robustness of quantum effects to decoherence and noise.

Aside from the fundamental interest, our model is also relevant for implementations of the QW where the imperfections arisen during the fabrication procedure alter the coupling constant between neighboring nodes (which could be implemented by waveguides or quantum dots), making it not perfectly known nor constant. We will focus on non-Gaussian noise, overcoming the widespread Gaussian approximation for classical noise [41]. Specifically, transition amplitudes will be perturbed by random telegraph noise (RTN) which, depending on the value of the autocorrelation time, will allow us to identify two very different dynamical behaviors for the walker. Moreover, we will consider the effect of different initial conditions, specifically an initial localized state and a Gaussian wave packet with nonzero initial velocity, in order to analyze both diffusion and transport phenomena on the lattice. In turn, analyzing the dynamical behavior of the particle in the different working regimes is extremely relevant in the context of reservoir engineering, as well as for noise characterization schemes [42-46].
Under the influence of noise, the walker should be described as an open quantum system, whose nonunitary evolution is influenced by an external complex environment. In this context, addressing the memory effects of the environment becomes a crucial issue, both from a fundamental and an information-theoretic point of view. We thus complete our analysis on noisy CTQWs by connecting their dynamical behavior with the non-Markovian (Markovian) character of the dynamical map. The concept of non-Markovianity of a quantum evolution has been discussed in terms of different analytic properties of the corresponding dynamical map [4753]. The common aim of these proposals is that to capture, possibly in a quantitative way, the physical mechanism making memory effects relevant for a physical system. As such, non-Markovianity is often a useful resource in quantum information processing [54-59] and our results goes in the same direction.

As a matter of fact, different definitions and quantifiers of non-Markovianity of a quantum map have been proposed [47-53], many of which require an optimization procedure which is not always feasible for systems with a large dimensionality. The general idea behind the concept of quantum non-Markovianity is that the environment has memory that breaks the time divisibility of the dynamical map and allows information to go back to the quantum system, e.g., recovering part of its lost coherence [60-63]. The non-Markovian character of coined QWs has been the subject of some attention [64-66], whereas the continuous-time case received little attention, even in the classical case [67].

Here, we observe that the emergence of two different (asymptotic) dynamical behaviors for the walker subject to non-Gaussian noise is linked to the presence of memory effects in the environment. Indeed, we show that there exists a connection between the autocorrelation time of the noise and the non-Markovian character of the dynamical map. Due to the difficulty in computing exactly the non-Markovianity of the evolution, we prove it in the presence of slow noise, whereas for fast noise we may offer a conjecture, based on repeated numerical experiments, about the Markovian character of the corresponding map.

The paper is structured as follows: In Sec. I, we introduce the model for a quantum walk on a noisy lattice, with stochastic tunneling amplitudes. In Sec. II, we present our results on the dynamics of the walker in the presence of noise and discuss the role of the different noise parameters. In Sec. III, we address the problem of determining the Markovian or non-Markovian character of the dynamical map, whereas Sec. IV closes the paper with some concluding remarks.

\section{MODEL}

In this section, we introduce the model for a CTQW on a one-dimensional lattice in the presence of noise. The physical situation we want to describe is an implementation of the QW where, due to imperfections arisen during the fabrication procedure of the lattice (such as an array of waveguide), the coupling constant between neighboring nodes is not perfectly known nor constant. Specifically, we describe these fabrication imperfections as stochastic time-dependent terms in the offdiagonal elements of the Hamiltonian. The global Hamiltonian 
may thus be effectively written as

$$
H(t)=H_{0}+V(t),
$$

where $H_{0}$ is the unperturbed Hamiltonian in Eq. (1), which we rewrite as

$$
H_{0}=\epsilon \mathbb{I}-\sum_{j}(|j\rangle\langle j+1|+| j+1\rangle\langle j|),
$$

$\epsilon$ being the onsite energy and $\mathbb{I}=\sum|j\rangle\langle j|$ the identity operator. The noise contribution is described by

$$
V(t)=v \sum_{j} g_{j}(t)(|j\rangle\langle j+1|+| j+1\rangle\langle j|),
$$

where the coefficients $\left\{g_{j}(t)\right\}$ represent the time-dependent fluctuations of the tunneling amplitudes between adjacent sites of the lattices, and $v$ denotes the coupling constant between the walker and an external environment generating the noise. Clearly, the two matrices do not commute $\left[H_{0}, V(t)\right] \neq 0$. As a consequence, they do not share a common set of eigenvectors and Eqs. (2) and (3) are not valid in the case of noisy QWs.

The evolved density matrix of the particle is the ensemble average

$$
\rho(t)=\left\langle U(t) \rho_{0} U^{\dagger}(t)\right\rangle_{\left\{g_{j}(t)\right\}},
$$

where $\langle\ldots\rangle_{\left\{g_{j}(t)\right\}}$ denotes the average taken over all possible realizations of the (independent) stochastic processes $\left\{g_{j}(t)\right\}$ and $U(t)$ is the unitary evolution operator

$$
U(t)=T \exp \left\{-i \int_{0}^{t} d s H(s)\right\},
$$

where $T$ denotes the time-ordering operator.

The noise coefficients $\left\{g_{j}(t)\right\}$ are stochastic classical processes whose features describe different kinds of lattice defects and, in turn, determine different dynamical behaviors of the walker. In this work, we will focus on independent stationary processes with autocorrelation function

$$
C(t)=\left\langle g_{j}(t) g_{k}(0)\right\rangle=\delta_{j k} \chi(t) .
$$

In particular, we focus on non-Gaussian processes and consider random telegraph noise, i.e., we describe the $\left\{g_{j}(t)\right\}$ as dichotomic variables which can switch between two values $\pm a$ with a certain switching rate $\gamma$ [68-72]. The parameter $a$ defines the strength or amplitude of the noise whereas $\gamma$ determines its time scale. The probability for the fluctuator $g_{j}$ to switch $n$ times after a time $t$ follows a Poisson distribution

$$
p_{n}(t)=\frac{(\gamma t)^{n}}{n !} e^{-\gamma t} .
$$

The autocorrelation function for the process is

$$
\chi(t)=a^{2} e^{-2 \gamma t},
$$

corresponding to a Lorentzian spectrum.

\section{Non-Markovianity of the dynamical map}

The quantum map in Eq. (7) may give rise to either a Markovian or non-Markovian evolution, depending on whether the memory effects of the environment are negligible or they influence the walker's dynamics. The non-Markovian character of the quantum evolution may be detected by looking at violations of equality [73]

$$
T\left(t_{2}, t_{0}\right)=T\left(t_{2}, t_{1}\right) T\left(t_{1}, t_{0}\right)
$$

for some triple $t_{2}>t_{1}>t_{0}$, where $T$ is a universal dynamical map defined by Eq. (7), i.e.,

$$
T\left(t_{b}, t_{a}\right) \rho\left(t_{a}\right) \equiv \rho\left(t_{b}\right)=\left\langle U\left(t_{b}, t_{a}\right) \rho\left(t_{a}\right) U^{\dagger}\left(t_{b}, t_{a}\right)\right\rangle_{\left\{g_{j}\right\}},
$$

where $\rho\left(t_{a}\right)$ is an arbitrary initial state of the system and $t_{b}>t_{a}$. Whenever the dynamics may be written as a composition of two maps as in Eq. (10), memory effects are not present and the evolution of the walker does not depend on its past. On the contrary, any violation of Eq. (10) provides evidence that the future evolution depends upon all its past history, i.e., the dynamics is non-Markovian. In this case, in fact, given two final states $\rho^{\prime}\left(t_{2}\right)=T\left(t_{2}, t_{0}\right) \rho\left(t_{0}\right)$ and $\rho\left(t_{2}\right)=$ $T\left(t_{2}, t_{1}\right) T\left(t_{1}, t_{0}\right) \rho\left(t_{0}\right)$, there exists an initial state for the system $\rho\left(t_{0}\right)$ such that $\rho\left(t_{2}\right) \neq \rho^{\prime}\left(t_{2}\right)$. A nonzero distance, e.g., a nonzero trace distance, between the final states $\rho\left(t_{2}\right)$ and $\rho^{\prime}\left(t_{2}\right)$ can be taken as an evidence of non-Markovianity for quantum systems subject to classical noise. To support our results on the non-Markovian character of the map, we also look for revivals in the trace distance between a pair of initial states subject to the same evolution, interpreted as a signature of information backflow into the system, according to Breuer-Laine-Piilo (BLP) measure [47]. The trace distance between two quantum states $\rho_{1}$ and $\rho_{2}$ is defined as

$$
D\left(\rho_{1}, \rho_{2}\right)=\frac{1}{2}\left\|\rho_{1}-\rho_{2}\right\|,
$$

where $\|A\|=\operatorname{Tr}\left[\sqrt{A^{\dagger} A}\right]$. Whenever $D$ has a monotonic behavior in time, the evolution is Markovian, otherwise if it oscillates in time, the quantum map is non-Markovian.

\section{DYNAMICS OF NOISY CONTINUOUS-TIME QUANTUM WALKS}

In this section, we present and discuss our results about the dynamics of CTQW in the presence of classical noise, mimicking disorder and/or defects in the lattice. The time evolution of the particle, described by Eq. (7), cannot be computed analytically for a large number of nodes, so we evaluated the ensemble averages numerically after Monte Carlo generating the values of the switching times.

In order to gain insight into the transition from quantum to classical behavior of the walker, we study the dynamics of various quantities. In particular, we analyze the evolution of the probability distribution of the particle over the lattice sites using the corresponding negentropy $N_{E}(t)$, the variance $\sigma^{2}(t)$ of the particle position, and the coherence $C(t)$ of the density matrix $\rho(t)$ as functions of time.

The probability distribution over the lattice site corresponds to the diagonal elements $\rho_{j j}(t)$ of the density matrix (7), while the variance is computed as $\sigma^{2}=\left\langle x^{2}\right\rangle-\langle x\rangle^{2}$, where $\left\langle x^{k}\right\rangle=\sum_{n} n^{k} \rho_{n n}$. The negentropy $N_{E}$ [74] is a measure of the non-Gaussianity of a probability distribution, i.e., it tells how much a probability distribution deviates from a normal distribution. The negentropy $N_{E}(Y)$ of a random variable $Y$ with distribution $p(y)$ is defined as difference between the Shannon entropy $H\left(Y_{G}\right)=-\sum_{y_{G}} p\left(y_{G}\right) \ln p\left(y_{G}\right)$ of a 

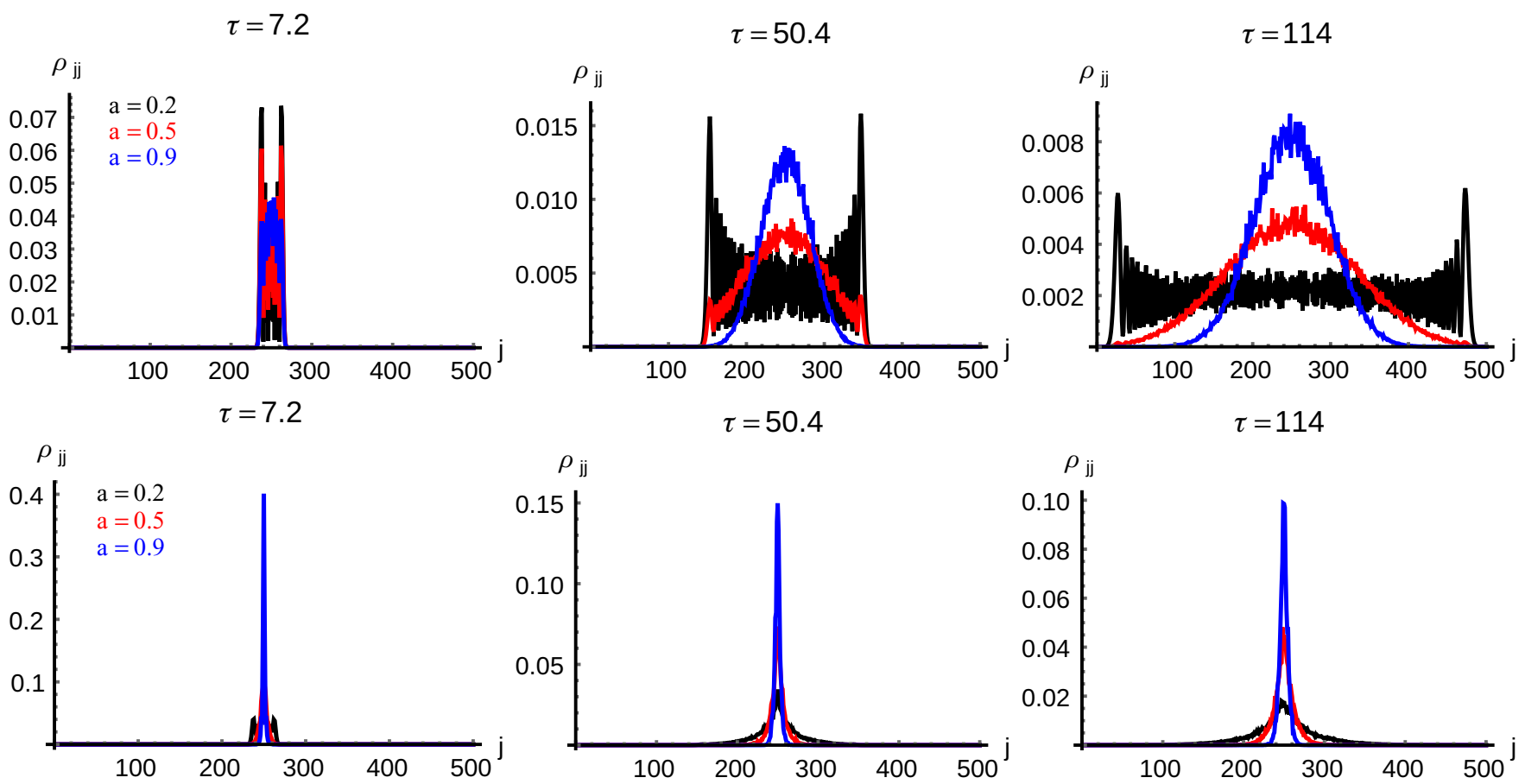

FIG. 1. Probability distribution of the particle over the lattice nodes at three different values of the interaction time $\tau$, for a ring lattice of $N=500$ sites, subject to RTN with $\gamma=10$ (upper row) and $\gamma=0.01$ (lower row). Different values of the noise amplitude $a=0.2,0.5$, and 0.9 are represented by the black, the red (light gray), and the blue (dark gray) lines, respectively. The particle is initially in the localized state $|N / 2\rangle$.

Gaussian random variable $Y_{G}$ with the same variance of $Y$ and the Shannon entropy $H(Y)$ of $Y$

$$
\begin{aligned}
N_{E}(Y) & =H\left(Y_{G}\right)-H(Y) \\
& =\frac{1}{2}\left[1+\ln \left(2 \pi \sigma_{y}^{2}\right)\right]+\sum_{y} p(y) \ln [p(y)],
\end{aligned}
$$

where $\sigma_{y}^{2}$ is the variance of both variables $Y_{G}$ and $Y . N_{E}$ is always a positive quantity, unless $Y$ is Gaussian (in this case $\left.N_{E}=0\right)$.

Finally, the coherence of a quantum state [75] is defined as the sum of the absolute values of the coherences of $\rho(t)$ :

$$
C(t)=\sum_{k, j, k \neq j}\left|\rho_{k j}(t)\right| .
$$

In order to study the effect of noise on the dynamics of the walker, different initial conditions have been considered, including both the case of a state $\left|\psi_{0}\right\rangle=\left|j_{0}\right\rangle$ initially localized on a single node of the lattice, and of a Gaussian wave packet with a certain width $\Delta$ and an initial nonzero velocity. The tunable parameters which we can change in order to obtain different dynamical evolutions for the walker are the amplitudes of the noise terms $g_{j}(t)$, which at every instant of time take values $\pm a$, and the switching rate $\gamma$.

In order to simplify the analysis of the dynamics, we exploit a scaling property of the system (with respect to the coupling v) and introduce the dimensionless time and the dimensionless switching rate as

$$
t \rightarrow \nu t \equiv \tau, \quad \gamma \rightarrow \gamma / \nu .
$$

\section{A. Localized initial state}

Let us first focus to the case where the particle is initially localized on the central lattice site $\left|\psi_{0}\right\rangle=|N / 2\rangle$. Figure 1 shows the probability distribution of the particle over the lattice sites at three different interaction times $\tau$ for selected noise amplitudes $a$ in the case fast noise $\gamma=10$ (upper row) and slow noise $\gamma=0.01$ (lower row). The two chosen values for the switching rates are good representatives of the two regimes of the RTN with fast $(\gamma \gg 1)$ and slow $(\gamma \ll 1)$ decaying autocorrelation function. In particular, a large value of $\gamma$ corresponds to a situation where the bistable fluctuators flip almost at every time step (remember that the average number of switches in a time interval $d t$ is $n=\gamma d t$ ), while RTN for very small values of the switching rate can be considered an example of quasistatic (but still random) noise.

The first fact emerging from Fig. 1 is that the two different regimes give rise to very different behaviors. Under the action of fast RTN, the walker spreads over the lattice with a probability distribution dependent on the noise strength $a$. The higher is the noise amplitude $a$, the stronger is the impact of defects on the dynamics of the walker. A transition from quantum to classical is induced over time. The probability distribution of the unperturbed walker, with the two peaks moving away from the starting node, is lost as the value of $a$ is increased: While for small values of the noise amplitude the typical quantum behavior is still present during the time evolution, for larger values of $a$ the interference pattern is completely lost already at small interaction times, and a Gaussian-type distribution centered around the initial position arises for large times $\tau$. The two tail peaks are suppressed while the central part of the distribution grows, as the value 

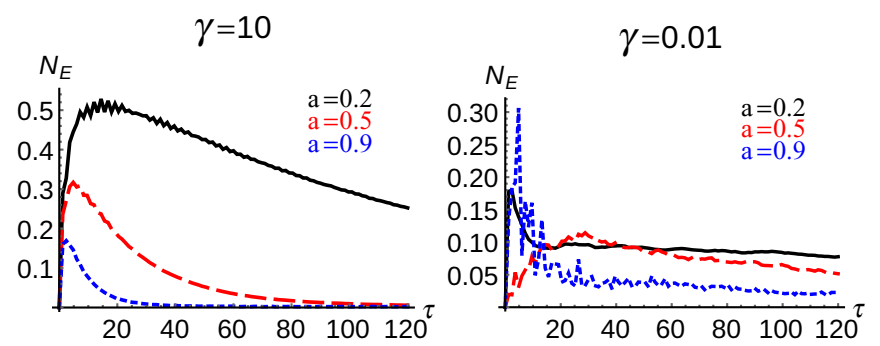

FIG. 2. Negentropy (12) of the probability distribution of the particle over the lattice nodes as a function of the interaction time $\tau$ for different values of the noise amplitude $a=0.2$ (solid black line), 0.5 (dashed red line), and 0.9 (dotted blue line), in the fast (left plot) and slow (right plot) regimes, computed for a QW on a 500-site lattice.

of $a$ is increased. The situation is very different in the case of a slow noise: the doubly peaked distribution vanishes with increasing noise amplitudes, but the probability distribution remains localized around its initial position. This effect is the dynamical counterpart of Anderson localization [28], already found for static noise affecting the diagonal terms of the Hamiltonian [36,76,77].

What emerges from our analysis, so far, is that in the fast noise regime [also referred to as the weak coupling regime since we are working with rescaled parameters, see Eq. (14)] we see a transition from quantum to classical diffusive behavior, while in the slow noise regime (strong coupling) we observe localization of the walker over few lattice sites. Since a classical random walk has a Gaussian probability distribution over the line, we may quantify the degree of classicality of the QW evolution by computing the negentropy (12), i.e., by looking how far the QW distribution is from a normal one. In Fig. 2, we report the behavior of $N_{E}$ as a function of time $\tau$ for different values of the noise amplitude $a$ in the case of a QW subject to fast RTN (left plot) and slow RTN (right plot). The two regimes for the autocorrelation function indeed identify two different behaviors for the $N_{E}$ : in the fast noise limit, the negentropy is smaller for long time as the noise strength is increased, indicating that a transition toward a classical, Gaussian probability distribution is induced by strong noise. On the other hand, in the slow noise regime, the negentropy, after an initial dynamics which is related to the noise amplitude, becomes almost constant, indicating that the probability distribution over the lattice changes only slightly as time is increased.

The effect of noise on the dynamics of the walker and the appearance of a phase transition may be analyzed in more details by looking at the time dependency of the variance $\sigma^{2}(\tau)$ of the position of the walker, i.e., the spread of the particle over the lattice. The first plot in Fig. 3 shows the variance for different values of the noise amplitude in the case of $\gamma=10$. For small times, the variance is quadratic in time, and it becomes linear at later times, a signature that a transition between quantum and classical diffusion has happened. In fact, the curves may easily be fitted by a quadratic function for interaction times below a given threshold $\tau<\tau_{c}$ and by a linear one above this threshold. The transition time $\tau_{c}$ depends upon the value of the noise amplitude and it is larger for smaller
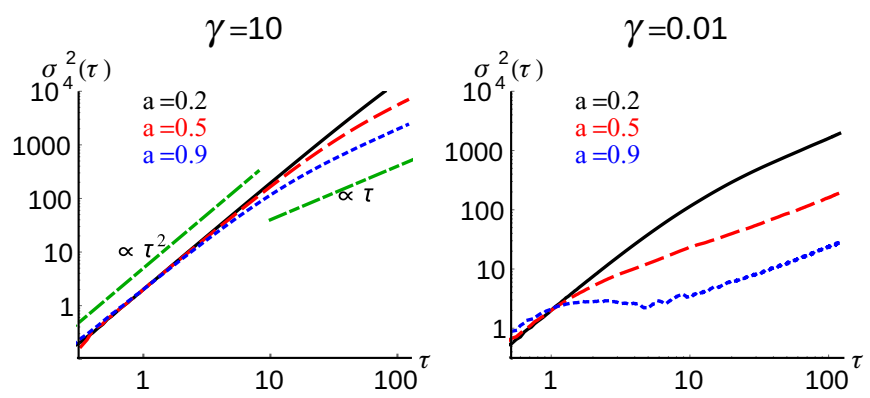

FIG. 3. Variance $\sigma^{2}(\tau)=\left\langle x^{2}\right\rangle-\langle x\rangle^{2}$ of the particle position as a function of the interaction time $\tau$, for a lattice of $N=500$ sites, subject to RTN with $\gamma=10$ (left plot) and $\gamma=0.01$ (right plot) for different values of the noise amplitude $a=0.2$ (solid black line), 0.5 (dashed red line), and 0.9 (dotted blue line). As a guide for the eyes, the green dotted-dashed curves indicate the different slopes in the left plot. The particle is initially in the localized state $|N / 2\rangle$.

value of $a$. Notice that in our system we have decoherence without dissipation, such that the position variance provides a good indicator of the transition. A different approach has been discussed for dissipative systems [78].

The second plot shows the same quantity but for $\gamma=0.01$. While for small noise amplitudes the walker can still propagate, as the noise strength increases, the variance becomes linear, indicating that the walker is diffusing very slowly through the lattice, thus confirming localization over few nodes around the initial site.

The analysis of the dynamics of distribution over the lattice sites, as well as those of the negentropy and of the variance, only involves the diagonal elements of the density matrix. In order to gain more insight into the behavior of the system we study the time evolution of the full density matrix by analyzing the dynamics of its coherence $C$, as defined in Eq. (13). Figure 4 shows the dynamics of coherence for a noisy CTQW, in the two regimes of fast (left plot) and slow (right plot) noises, for different values of the noise amplitude $a$. In the fast regime, the dependency on $a$ is clearly evident: the off-diagonal elements of the density matrix grow over time (here for a fixed interaction time $\tau=120$ ) for small values of the noise amplitude. If we increase the value of $a, C(\tau)$ starts decreasing after an initial growth, a sign of
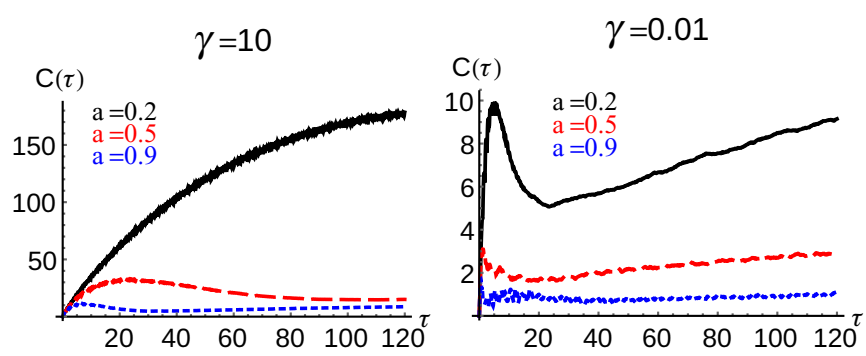

FIG. 4. The coherence $C$ of Eq. (13) as a function of the interaction time $\tau$ for different values of the noise amplitude $a=$ 0.2 (solid black line), 0.5 (dashed red line), and 0.9 (dotted blue line), in the fast (left plot) and slow (right plot) regimes, computed for a QW on a lattice with $N=500$ sites. 

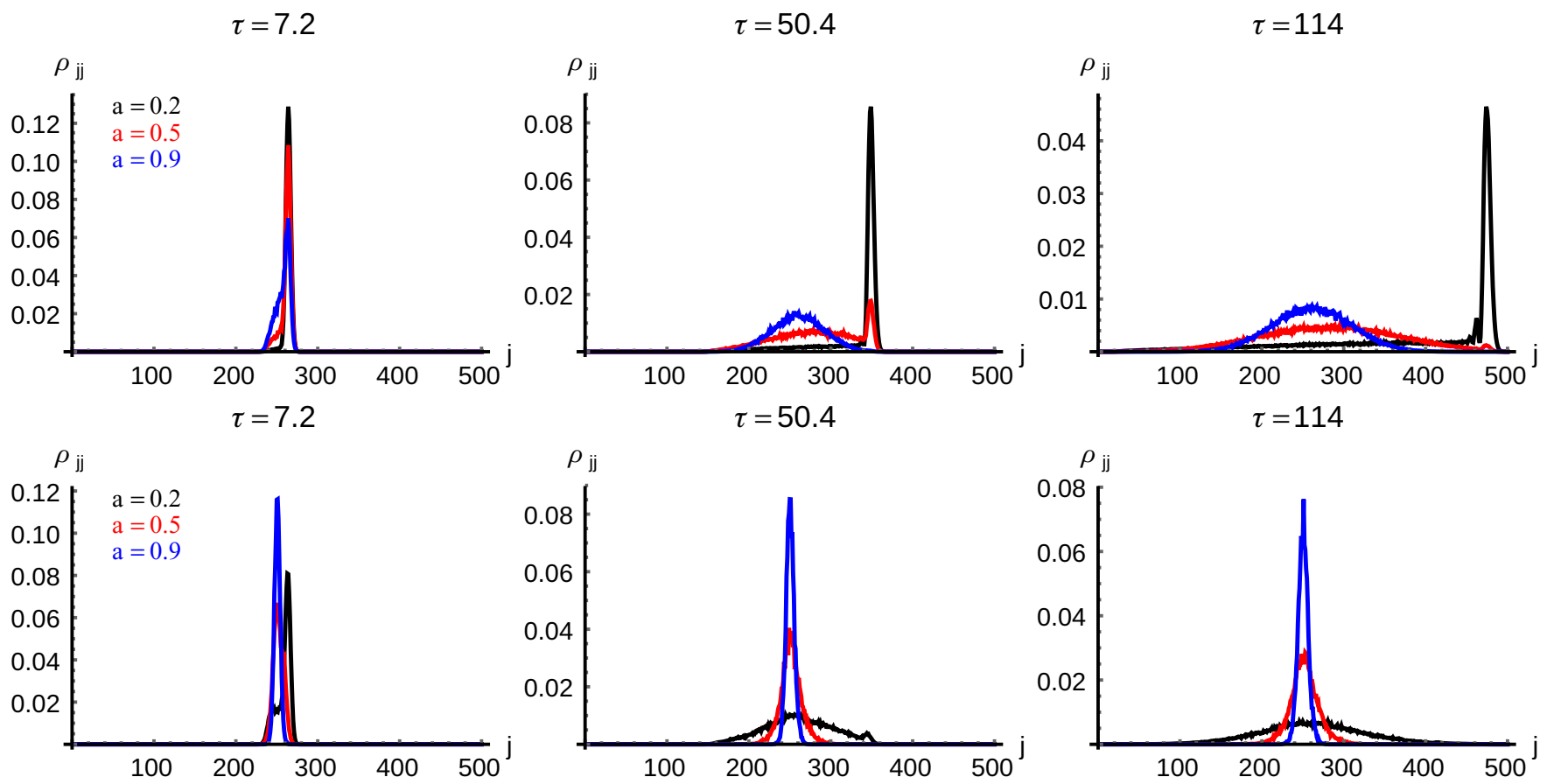

FIG. 5. Probability distribution of the particle over the lattice nodes, for different values of the noise amplitude $a=0.2$ (black line), 0.5 [red (light gray) line], and 0.9 [blue (dark gray) line] and interaction time $\tau$, for a lattice of $N=500$ sites, subject to RTN with $\gamma=10$ (upper row) and $\gamma=0.01$ (lower row). The particle is initially described as a Gaussian wave packet with $\Delta=3$ and initial velocity $k_{0}=\frac{3 \pi}{2}$.

decoherence induced by noise. In the slow noise regime (right panel), the coherence $C(\tau)$ initially increases and then drop to a constant for large values of $a$, while regrowth is seen for small noise amplitudes. This indicates the survival of quantumness over time, as expected for a system undergoing Anderson localization. The initial increase of $C(\tau)$ is larger for smaller values of the noise amplitude $a$. We also point out that the magnitude of $C(\tau)$ for slow noise is always below the corresponding values for fast noise, in agreement with the fact that the initially localized state tends to spread very little over the lattice nodes.

So far, we have shown that for a CTQW propagating in a disordered lattice subject to RTN, two main typical long-time behaviors arise: the walker can spread very slowly, staying localized over a small fraction of the total number of sites, or it can propagate through the graph with a standard deviation proportional to the square root of time. One may wonder whether this features depends upon the choice of the localized initial condition, or they are more general characteristic of noisy QWs.

\section{B. An initial Gaussian wave packet}

In order to better analyze the effect of dynamical disorder on the quantum walk, we consider a different initial condition: instead of a localized initial state, we study the case of an initial Gaussian wave packet with a nonzero dimensionless velocity $k_{0}$ (in unit of $\hbar$ ) and dimensionless standard deviation $\Delta$, such that the initial (pure) state $\left|\psi_{G}\right\rangle$ may be written as

$$
\left|\psi_{G}\right\rangle=\sum_{j=1}^{N}\left(\frac{1}{\sqrt{2 \pi \Delta^{2}}} e^{-\frac{\left(j-\frac{N}{2}\right)^{2}}{2 \Delta^{2}}}\right)^{1 / 2} e^{-i k_{0} j}|j\rangle .
$$

This initial condition allows us to imprint an initial momentum distribution to the particle and investigate under which conditions transport phenomena over the graph is possible in the presence of noise. In Fig. 5, we report the probability distribution over the lattice sites for different times, for different values of the noise amplitude and the noise parameters, in analogy with Fig. 1. As before, we compare the dynamical behavior of the walker subject to fast and slow RTN. For small values of the parameter $a$, the wave packet moves away from its initial position during time, indeed indicating transport through the graph. The same features seen with a localized initial condition are found, thus indicating that the main features of the dynamics are imputable to decoherence and thus independent on the choice of the initial condition. Fast noise leads, indeed, to a Gaussian-type probability distribution as the noise amplitude becomes larger, while slow noise keeps the distribution localized.

Figure 6 shows the dynamical behaviors of the mean position $\langle x(\tau)\rangle$ of the particle, its variance $\sigma^{2}(\tau)$, and the coherence $C(\tau)$ in the two noise regimes. The main difference here with respect to the localized case is that the mean position $\langle x\rangle$ changes with time for fast noise, moving away from the initial position, indicating that not only diffusion is present, but also drift. From these results, we conclude that transport is possible if the strength of the noise $a$ is small, otherwise, the diffusive (or localized) behavior prevails, threatening the possibility of transport. This can also be confirmed by the analysis of the variance in the two regimes. As the noise strength is increased in the weak coupling regime, the effect of decoherence manifests through the spread of the wave packet over the nodes, i.e., $\sigma^{2}(\tau)$ increases rapidly in time. In the other regime, instead, we see again that as the value of $a$ is 

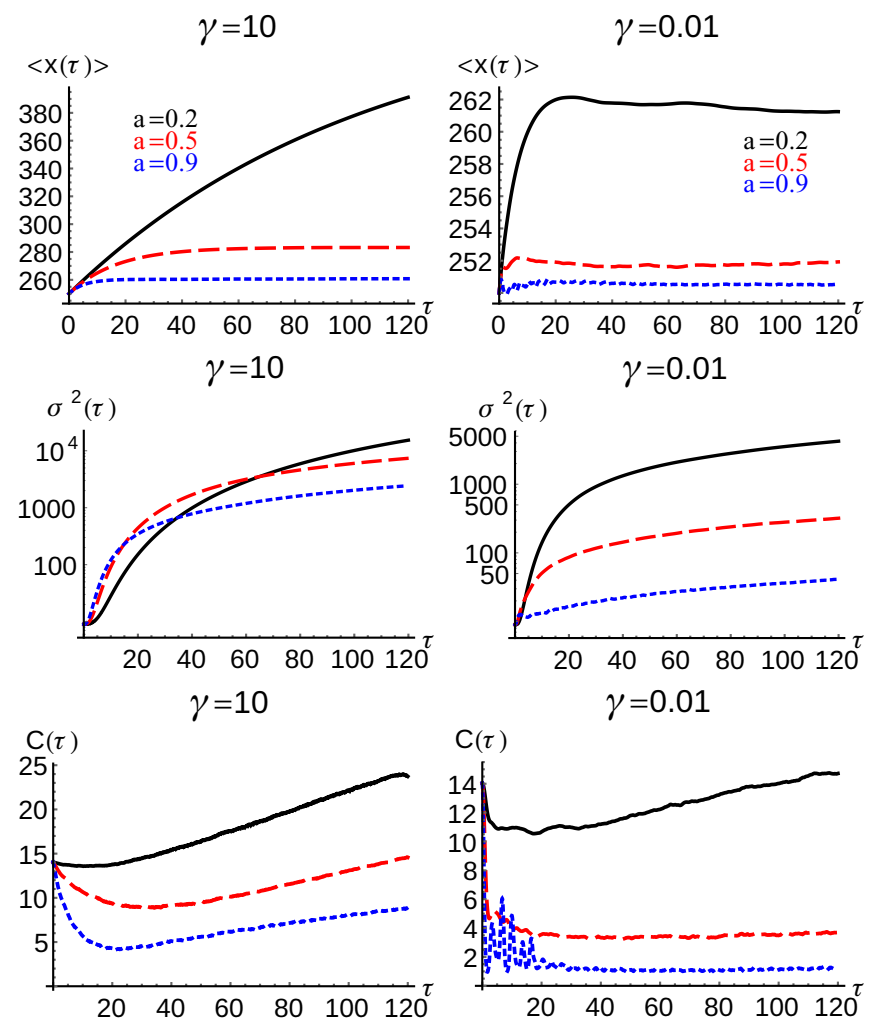

FIG. 6. From top to bottom: mean value of the particle position $\langle x(\tau)\rangle$, variance $\sigma^{2}(\tau)$, and coherence $C(\tau)$ as functions of the interaction time $\tau$, for different values of the noise amplitude $a=$ 0.2 (solid black line), 0.5 (dashed red line), and 0.9 (dotted blue line), for a ring lattice of 500 sites, subject to RTN with $\gamma=10$ (left column) and $\gamma=0.01$ (right column). The particle is initially described as a Gaussian wave packet with $\Delta=3$ and initial velocity $k_{0}=\frac{3 \pi}{2}$.

increased, the wave function is localized with a slowly varying variance.

The coherence of a quantum state initially prepared in a superposition decays faster for larger values of $a$ in both regimes. However, for the explored values of the interaction time, superposition of states were preserved.

Our results indicate that the time scale of the noise, i.e., its autocorrelation function, determines the qualitative behavior of the walker dynamics over the lattice. In the next section, we are going to analyze and discuss how the two working regimes are related to the Markovian or non-Markovian nature of the quantum map describing the evolution of the walker.

\section{NON-MARKOVIANITY OF CTQWS}

Non-Markovian quantum dynamics arises when memory effects become relevant and the future evolution of a quantum system does not only depend upon its present, but it is instead determined by its full or partial past history.

A slowly decaying autocorrelation function for the environmental noise may be intuitively associated with memory effects in the environment, while fast decaying, deltalike, autocorrelation, is usually associated to Markovian dynamics. In order to check whether this connection is true, we assess the

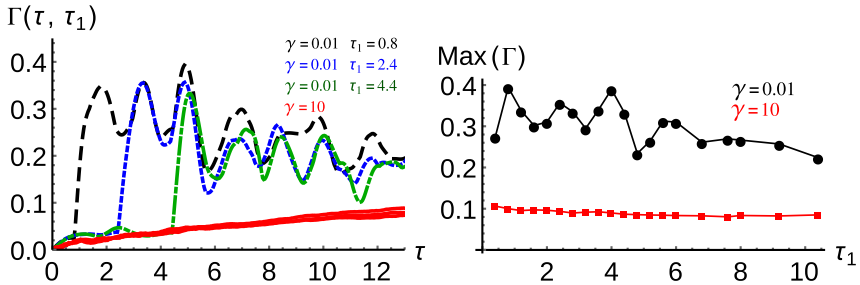

FIG. 7. Left: trace distance $\Gamma\left(\tau, \tau_{1}\right)$ as a function of the interaction time $\tau$ in the case of slow (dashed black, dotted blue, and dotteddashed green lines) and fast (solid red lines) noise. The different curves are for different values of intermediate time $\tau_{1}$ in Eq. (16). The particle is initially in a localized state. Right: maximum of $\Gamma\left(\tau, \tau_{1}\right)$ over time $\tau$ as a function of $\tau_{1}$. The black points are for $\gamma=0.01$ and the red squares for $\gamma=10$.

non-Markovian character of the dynamical map by looking at violation of Eq. (10) by some given initial states, and also by studying the evolution of the trace distance between suitably chosen pairs of states. Both techniques have limits, i.e., may not provide full characterization of the dynamical map, since for CTQWs one cannot span all intermediate times $\tau_{1}$ to check validity of Eq. (10) or span the full Hilbert space in looking for states that experience information backflow. Yet, we may obtain numerical evidence [violation of equality (10)] for the non-Markovianity of the evolution in the presence of slow noise.

In order to prove the violation of Eq. (10), we consider a suitable initial state $\rho_{0}$ and evaluate the trace distance (11) between the state obtained by applying the full map $\rho(\tau)=$ $T(\tau, 0) \rho_{0}$ and the state resulting from the composition $\rho^{\prime}(\tau)=$ $T\left(\tau, \tau_{1}\right) T\left(\tau_{1}, 0\right) \rho_{0}$, i.e.,

$$
\Gamma\left(\tau, \tau_{1}\right)=D\left[T(\tau, 0) \rho_{0}, T\left(\tau, \tau_{1}\right) T\left(\tau_{1}, 0\right) \rho_{0}\right] .
$$

In the left panel of Fig. 7, we show the behavior of $\Gamma\left(\tau, \tau_{1}\right)$ as a function of time in the fast and slow noise regimes, starting from a localized initial condition. For a given value of $\gamma$, the different lines correspond to different values of the intermediate time $\tau_{1}$ in Eq. (16). As it is apparent from the plot, in the fast noise regime $\Gamma\left(\tau, \tau_{1}\right)$ is close to zero at any time and for any choice of the intermediate $\tau_{1}$, i.e., no differences appear between the full and composed dynamical maps. The fact that the trace distance is not strictly zero is imputable to the accumulation of numerical noise since we are averaging over a finite number of realizations of the stochastic processes $\left\{g_{j}(t)\right\}$ and not performing the true ensemble average as in Eq. (7).

On the contrary, strong differences in the dynamics are revealed when we consider the slow noise regime [i.e., strong coupling, see Eq. (14)], indicating that the dynamical map involves memory effects for slowly fluctuating environments with long-lasting correlations. This is confirmed by the results reported in the right panel of Fig. 7, which shows the maximum of the trace distance $\Gamma$ over time, for different values of $\tau_{1}$, in both the fast and slow noise regimes. When the dynamical evolution is split at a certain value of $\tau_{1}$ in the slow noise regime, the difference between the full and composed dynamical maps is apparent, while in the fast regime $(\gamma=10)$, 

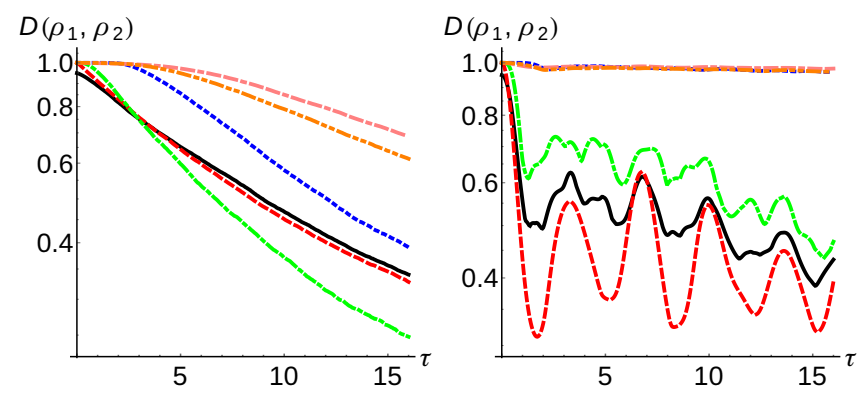

FIG. 8. Analysis of the trace distance for selected initial pairs of states. Trace distance for particle subject to RTN, for fast (left) and slow (right) noise. Different colors refer to different choices of the initial pairs of pure states: (solid black line) $\left|x_{0}\right\rangle,\left|\psi_{G}\left(x_{0}, k_{0}\right)\right\rangle$, (dashed red line) $\left.\left|\psi_{G}\left(x_{0}, k_{0}\right\rangle,\right| \psi_{G}\left(x_{0}, k_{0}+\frac{20 \pi}{N}\right)\right\rangle$, (dotted-dashed green line) $\left|x_{0}\right\rangle, \frac{\left|x_{0}+3\right\rangle+\left|x_{0}-3\right\rangle}{\sqrt{2}}$, (dotted blue line) $\left|x_{0}\right\rangle,\left|x_{0}+10\right\rangle$, (dotted-dasheddashed pink line) $\left.\left.\left|\psi_{G}\left(x_{0}, k_{0}\right\rangle,\right| \psi_{G}\left[x_{0}+20, k_{0}+\frac{20 \pi}{N}\right)\right]\right\rangle$, (dotteddotted-dashed orange line) $\left|x_{0}\right\rangle,\left|\psi_{G}\left(x_{0}+6, k_{0}\right)\right\rangle$, where $x_{0}=N / 2$, and $\mid \psi_{G}\left(x_{0}, k_{0}\right\rangle$ is the initial Gaussian wave packet in Eq. (15) with initial position $x_{0}$, velocity $k_{0}=3 \pi / 2$, and standard deviation $\Delta=4$.

values of the maximum are compatible with the numerical noise.

Notice that this is a property of the map, and thus it may not reveal itself for all initial states. Indeed, in our case, we have detected clear violation of Eq. (10) using localized initial conditions, while starting from an initial Gaussian wave packet (with or without a velocity distribution) lead to small value $\Gamma\left(\tau, \tau_{1}\right)$, i.e., to nearly divisible evolution.

On the other hand, the non-Markovian character of the dynamical map induced by slow noise is confirmed by analyzing the behavior of the trace distance between initial pairs of states, as required by BLP measure. Oscillations in time of $D\left[\rho_{1}(\tau), \rho_{2}(\tau)\right]$ for some given initial pair $\rho_{1}(0), \rho_{2}(0)$ provide evidence for information backflow to the quantum system from the surrounding environment. It is thus necessary and sufficient to find one initial pair for which the trace distance is nonmonotonic to prove that the dynamical map is non-Markovian, even if we cannot make a qualitative statement about the degree of non-Markovianity. Indeed, in the case of slow RTN, it is quite simple to sample the state space and find an initial pair of states for which the trace distance shows revivals during time evolution, as shown in Fig. 8. On the other hand, we could not find any initial pair leading to nonmonotonic behavior in the fast noise regime, a fact suggesting that the map may be Markovian, even though this does not prove it, since we are not able to check all possible initial states. Still, this result is in agreement with the analysis of the composition equality in (10), thus indicating the lack of memory effects in the fast noise regime and, in turn, provides strong indication in that direction. Overall, our results show that in the slow noise (strong coupling) regime, the dynamics is non-Markovian: memory effects are important and allow one to observe information backflow. At the same time, there are robust numerical evidences that the fast noise (weak coupling) regime corresponds to a Markovian dynamics. These results are also in agreement with our previous results for simpler systems, e.g., concerning the non-Markovianity of quantum maps describing the interaction of qubits with classical fluctuating fields [61].

\section{CONCLUSIONS}

In the last decade there have been several proposals to implement quantum walks in different systems and different kinds of lattices [40], also addressing scalability and feasibility in realistic conditions. In this framework, it becomes crucial to have more realistic theoretical models, which take into account the effects of noise and assess the residual quantumness of the systems. In fact, imperfections in the fabrication of the lattice may introduce randomness in the tunneling energy of the walker, thus inducing detrimental fluctuations that may, or may not, destroy the quantum effects in the system.

In this paper, we have studied in details the dynamics of noisy one-dimensional CTQWs. Defects and disorder in the lattice have been described as stochastic classical processes governing the off-diagonal elements of the Hamiltonian, in order to describe fluctuations in the tunneling amplitudes between neighboring sites. The walker dynamics has been then computed as an ensemble average over possible realizations of the noise. We found that, depending on the ratio between the autocorrelation time of the noise and the coupling between the walker and the external environment generating the noise, two different dynamical regimes appear.

If the walker is strongly coupled to its environment, the resulting slow noise confines the walker into few lattice nodes. On the contrary, a weakly coupled walker subject to fast noise is driven through a transition from quantum ballistic diffusion to a classical diffusive propagation over the lattice. The peculiar features of the two dynamical regimes have been confirmed by analyzing the variance of the particle position, the negentropy of the distribution, and the overall coherence of the full density matrix. We have analyzed different initial conditions for the walker, either localized or a Gaussian wave packet, thus also exploring the conditions under which we have information transfer through the lattice. We found that transport is possible if the amplitude $a$ of the noise is small, otherwise the diffusive (or localized) behavior prevails.

Upon analyzing the properties of the dynamical map, we have established a connection between the behavior of the walker in the slow noise (strong coupling) regime and the non-Markovian character of the evolution. In particular, we have shown that in this regime the dynamics cannot be written as the composition of two memoryless universal dynamical maps, i.e., violates Eq. (10), a signature that memory effects are important, and that the information lost because of noise may flow back to the system. In the fast noise (weak coupling) regime, numerical evidences strongly suggest the Markovianity of the quantum map, even if a conclusive proof is not currently available.

By tuning the ratio between the memory parameter of the noise and the coupling with the walker, it is possible to move continuously from one dynamical behavior to the other and observe the corresponding transition between the two dynamical phases (see Table I). This is a relevant feature since the chance of controlling the transition between different evolutions would serve as guidelines for reservoir engineering, 
TABLE I. Summary of the main features of two dynamical regimes.

\begin{tabular}{lcc}
\hline \hline Value of $\gamma$ & $\gamma \ll 1$ & $\gamma \gg 1$ \\
\hline $\begin{array}{l}\text { Regime } \\
\text { (coupling) }\end{array}$ & Slow noise & Fast noise \\
Dynamics & Localized & $\begin{array}{c}\text { Transition to classical } \\
\text { diffusion }\end{array}$ \\
Memory [see Eq. (10)] & Yes & No \\
BLP measure & Non-Markovian & Markovian \\
\hline \hline
\end{tabular}

where noise may be exploited to enhance some desired dynamical features.

\section{ACKNOWLEDGMENTS}

C.B. and M.G.A.P. thank D. Tamascelli and K. Luoma for discussions and useful suggestions. This work has been supported by EU through the Collaborative Projects QuProCS (Grant Agreement No. 641277), by UniMI through the H2020 Transition Grant No. 14-6-3008000-625, and by UNIMORE through FAR2014.
[1] J. Kempe, Contemp. Phys. 44, 307 (2003).

[2] S. E. Venegas-Andraca, Quant. Inf. Proc. 11, 1015 (2012).

[3] A. Ambainis, Int. J. Quantum. Inf. 01, 507 (2003).

[4] A. M. Childs, Phys. Rev. Lett. 102, 180501 (2009).

[5] O. Mülken and A. Blumen, Phys. Rep. 502, 37 (2011).

[6] O. Mülken, V. Bierbaum, and A. Blumen, J. Chem. Phys. 124, 124905 (2006).

[7] Z. Darázs, A. Anishchenko, T. Kiss, A. Blumen, and O. Mülken, Phys. Rev. E 90, 032113 (2014).

[8] M. Cho, H. M. Vaswani, T. Brixner, J. Stenger, and G. R. Fleming, J. Phys. Chem. B 109, 10542 (2005).

[9] A. Nazir, B. W. Lovett, S. D. Barrett, J. H. Reina, and G. A. D. Briggs, Phys. Rev. B 71, 045334 (2005).

[10] T. Odagaki and M. Lax, Phys. Rev. B 24, 5284 (1981).

[11] Y. Aharonov, L. Davidovich, and N. Zagury, Phys. Rev. A 48, 1687 (1993).

[12] E. Farhi and S. Gutmann, Phys. Rev. A 58, 915 (1998).

[13] B. C. Travaglione and G. J. Milburn, Phys. Rev. A 65, 032310 (2002).

[14] C. A. Ryan, M. Laforest, J. C. Boileau, and R. Laflamme, Phys. Rev. A 72, 062317 (2005).

[15] P. Xue, H. Qin, and B. Tang, Sci. Rep. 4, 4825 (2014).

[16] Q. Hao and X. Peng, Chin. Phys. B 23, 010301 (2014).

[17] H. B. Perets, Y. Lahini, F. Pozzi, M. Sorel, R. Morandotti, and Y. Silberberg, Phys. Rev. Lett. 100, 170506 (2008).

[18] J. Du, H. Li, X. Xu, M. Shi, J. Wu, X. Zhou, and R. Han, Phys. Rev. A 67, 042316 (2003).

[19] A. Patel, K. S. Raghunathan, and P. Rungta, Phys. Rev. A 71, 032347 (2005).

[20] F. W. Strauch, Phys. Rev. A 74, 030301(R) (2006).

[21] D. Ben-Avraham, E. M. Bollt, and C. Tamon, Quantum Inf. Proc. 3, 295 (2004).

[22] O. Mülken and A. Blumen, Phys. Rev. E 71, 036128 (2005).

[23] A. Schreiber, K. N. Cassemiro, V. Potoček, A. Gábris, I. Jex, and Ch. Silberhorn, Phys. Rev. Lett. 106, 180403 (2011).

[24] G. Di Giuseppe, L. Martin, A. Perez-Leija, R. Keil, F. Dreisow, S. Nolte, A. Szameit, A. F. Abouraddy, D. N. Christodoulides, and B. E. A. Saleh, Phys. Rev. Lett. 110, 150503 (2013).

[25] A. Crespi, R. Osellame, R. Ramponi, V. Giovannetti, R. Fazio, L. Sansoni, F. De Nicola, F. Sciarrino P. Mataloni, Nat. Photonics 7, 322 (2013).

[26] V. Kendon, Math. Struct. Comput. Sci. 17, 1169 (2006).

[27] L. Sapienza et al., Science 327, 1352 (2010).

[28] P. W. Anderson, Phys. Rev. 109, 1492 (1958).

[29] D. K. Wojcik and J. R. Dorfman, Phys. Rev. Lett. 90, 230602 (2003).
[30] M. Annabestani, S. J. Akhtarshenas, and M. R. Abolhassani, Phys. Rev. A 81, 032321 (2010).

[31] C. C. López and J. P. Paz, Phys. Rev. A 68, 052305 (2003).

[32] J. Košík, V. Bužek, and M. Hillery, Phys. Rev. A 74, 022310 (2006).

[33] A. Ahlbrecht, H. Vogts, A. H. Werner, and R. F. Werner, J. Math. Phys. 52, 042201 (2011).

[34] F. De Nicola, L. Sansoni, A. Crespi, R. Ramponi, R. Osellame, V. Giovannetti, R. Fazio, P. Mataloni, and F. Sciarrino, Phys. Rev. A 89, 032322 (2014).

[35] A. Alberti, W. Alt, R. Werner, and D. Meschede, New J. Phys. 16, 123052 (2014).

[36] J. P. Keating, N. Linden, J. C. F. Matthews, and A. Winter, Phys. Rev. A 76, 012315 (2007).

[37] Y. Yin, D. E. Katsanos, and S. N. Evangelou, Phys. Rev. A 77, 022302 (2008).

[38] J. A. Izaac, J. B. Wang, and Z. J. Li, Phys. Rev. A 88, 042334 (2013).

[39] D. de Falco and D. Tamascelli, J. Phys. A: Math. Theor. 46, 225301 (2013).

[40] J. Wang and K. Manouchehri, Physical Implementation of Quantum Walks (Springer, Berlin, 2014).

[41] E. Schwarzer and H. Haken, Phys. Lett. A 42, 317 (1972).

[42] G. A. Alvarez and D. Suter, Phys. Rev. Lett. 107, 230501 (2011)

[43] C. Benedetti, F. Buscemi, P. Bordone, and M. G. A. Paris, Phys. Rev. A 89, 032114 (2014).

[44] C. Benedetti and M. G. A. Paris, Phys. Lett. A 378, 2495 (2014).

[45] M. A. C. Rossi and M. G. A. Paris, Phys. Rev. A 92, 010302 (2015).

[46] A. Zwick, G. A. Alvarez, and G. Kurizki, Phys. Rev. Appl. 5, 014007 (2016).

[47] H. P. Breuer, E.-M. Laine, and J. Piilo, Phys. Rev. Lett. 103, 210401 (2009).

[48] R. Vasile, S. Maniscalco, M. G. A. Paris, H.-P. Breuer, and J. Piilo, Phys. Rev. A 84, 052118 (2011).

[49] M. M. Wolf, J. Eisert, T. S. Cubitt, and J. I. Cirac, Phys. Rev. Lett. 101, 150402 (2008).

[50] A. Rivas, S. F. Huelga, and M. B. Plenio, Phys. Rev. Lett. 105, 050403 (2010).

[51] X.-M. Lu, X. Wang, and C. P. Sun, Phys. Rev. A 82, 042103 (2010).

[52] S. Luo, S. Fu, and H. Song, Phys. Rev. A 86, 044101 (2012).

[53] S. Lorenzo, F. Plastina, and M. Paternostro, Phys. Rev. A 88, 020102 (2013).

[54] A. W. Chin, S. F. Huelga, and M. B. Plenio, Phys. Rev. Lett. 109, 233601 (2012). 
[55] R. Vasile, S. Olivares, M. G. A. Paris, and S. Maniscalco, Phys. Rev. A 83, 042321 (2011).

[56] E.-M. Laine, H.-P. Breuer, and J. Piilo, Sci. Rep. 4, 4620 (2014)

[57] S. F. Huelga, A. Rivas, and M. B. Plenio, Phys. Rev. Lett. 108, 160402 (2012).

[58] B. Bylicka, D. Chruściński, and S. Maniscalco, Sci. Rep. 4, 5720 (2014).

[59] D. Chruściński and S. Maniscalco, Phys. Rev. Lett. 112, 120404 (2014).

[60] R. Lo Franco, B. Bellomo, S. Maniscalco, and G. Compagno, Int. J. Mod. Phys. B 27, 1345053 (2013).

[61] C. Benedetti, M. G. A. Paris, and S. Maniscalco, Phys. Rev. A 89, 012114 (2014).

[62] P.-W. Chen and M. M. Ali, Sci. Rep. 4, 6165 (2014).

[63] A. Orieux, A. D’ Arrigo, G. Ferranti, R. Lo Franco, G. Benenti, E. Paladino, G. Falci, F. Sciarrino, and P. Mataloni, Sci. Rep. 5, 8575 (2015).

[64] X. Peng and Z. Yong-Sheng, Chin. Phys. B 22, 070302 (2013).

[65] M. Hinarejos, C. Di Franco, A. Romanelli, and A. Pérez, Phys. Rev. A 89, 052330 (2014).
[66] K. Luoma and J. Piilo, arXiv:1509.04231.

[67] J.-H. Jeon, E. Barkai, and R. Metzler, J. Chem. Phys. 139, 121916 (2013).

[68] B. Abel and F. Marquardt, Phys. Rev. B 78, 201302(R) (2008).

[69] D. Zhou, A. Lang, and R. Joynt, Quantum Inf. Proc. 9, 727 (2010).

[70] P. Bordone, F. Buscemi, and C. Benedetti, Fluct. Noise Lett. 11, 1242003 (2012).

[71] C. Benedetti, F. Buscemi, P. Bordone, and M. G. A. Paris, Int. J. Quantum. Inf. 10, 1241005 (2012).

[72] C. Benedetti, F. Buscemi, P. Bordone, and M. G. A. Paris, Phys. Rev. A 87, 052328 (2013).

[73] S. C. Hou, S. L. Liang, and X. X. Yi, Phys. Rev. A 91, 012109 (2015).

[74] P. Comon, Signal Process. 36, 287 (1994).

[75] T. Baumgratz, M. Cramer, and M. B. Plenio, Phys. Rev. Lett. 113, 140401 (2014).

[76] Z. J. Li, J. A. Izaac, and J. B. Wang, Phys. Rev. A 87, 012314 (2013).

[77] J. Ghosh, Phys. Rev. A 89, 022309 (2014).

[78] S. Garnerone, Phys. Rev. A 86, 032342 (2012). 